\title{
灵国pubvet
}

https://doi.org/10.31533/pubvet.v15n06a846.1-8

\section{Análise retrospectiva sobre leptospirose na região metropolitana I do Rio de Janeiro de 2015 a 2019}

\author{
Thayná de Amorim Santos ${ }^{1 *}$, Alexandre de Pina Costa ${ }^{2 *} \bullet$ \\ ${ }^{1}$ Graduanda em Medicina Veterinária pela Universidade do Grande Rio, Campus I. Duque de Caxias - RJ, Brasil. \\ ${ }^{2}$ Professor adjunto do curso de Medicina Veterinária da Universidade do Grande Rio, Campus I. Duque de Caxias - RJ, Brasil. \\ *Autor para correspondência, E-mail: thaynadeamorim@unigranrio.br
}

Resumo. O trabalho teve como objetivo analisar os 438 casos notificados através do Sistema de Informação de Agravos de Notificação (SINAN) e definir um perfil epidemiológico da leptospirose na região metropolitana I do estado do Rio de Janeiro entre os anos de 2015 a 2019. A leptospirose é uma zoonose de notificação obrigatória e de suma importância para a saúde pública devido a elevada incidência de novos casos e pelo seu alto grau de transmissão, principalmente durante períodos de enchentes. Os resultados obtidos através da plataforma DATASUS-TABNET confirmaram a maior prevalência de casos positivos da doença na cidade do Rio de Janeiro e nos municípios da Baixada Fluminense, além disso, observou-se o maior acometimento de indivíduos do gênero masculino, de faixa etária entre 20 a 59 anos e que possuíam um baixo grau de escolaridade, foi verificado um alto percentual de cura nos acometidos, indicando uma melhora considerável desses pacientes. Pôde-se concluir que a prevalência da leptospirose está intimamente relacionada a fatores socioeconômicos como saneamento básico, acesso à informação e uma adequada infraestrutura no local de residência dos acometidos.

Palavras-chave: Leptospirose, região metropolitana, saúde pública, zoonose

\section{Retrospective analysis of leptospirosis in metropolitan region I of Rio de Janeiro from 2015 to 2019}

\begin{abstract}
The article aimed to analyze the 438 cases reported through the Notifiable Diseases Information System (SINAN) and define an epidemiological profile of leptospirosis in metropolitan region I of the state of Rio de Janeiro between the years 2015 to 2019. Leptospirosis is a zoonosis of mandatory reporting and of paramount importance for public health due to the high incidence of new cases and its high degree of transmission, especially during periods of flooding. The results obtained through the DATASUSTABNET platform confirmed the higher prevalence of positive cases of the disease in the city of Rio de Janeiro and in the cities of Baixada Fluminense, in addition, there was a greater involvement of male individuals, aged between 20 to 59 years old and who had a low level of education, a high percentage of cure was found in those affected, indicating a considerable improvement in these patients. It was concluded that the prevalence of leptospirosis is closely related to socioeconomic factors such as basic sanitation, access to information and an adequate infrastructure in the place of residence of the affected people.
\end{abstract}

Keywords: Leptospirosis, metropolitan region, public health, zoonosis 


\title{
Análisis retrospectivo de la leptospirosis en la Región Metropolitana I de Río de Janeiro de 2015 a 2019
}

\begin{abstract}
Resumen. El objetivo de este trabajo fue analizar los 438 casos notificados a través del Sistema de Información de Enfermedades Notificables (SINAN) y definir un perfil epidemiológico de la leptospirosis en la región metropolitana I del estado de Río de Janeiro entre los años 2015 a 2019. La leptospirosis es una zoonosis de notificación obligatoria y de suma importancia para la salud pública debido a la alta incidencia de nuevos casos y su alto grado de transmisión, especialmente durante los períodos de inundaciones. Los resultados obtenidos a través de la plataforma DATASUS-TABNET confirmaron la mayor prevalencia de casos positivos de la enfermedad en la ciudad de Río de Janeiro y en los municipios de Baixada Fluminense, además, hubo una mayor participación de individuos del sexo masculino, con edades entre De 20 a 59 años y que tenían un bajo nivel educativo, se encontró un alto porcentaje de curación en los afectados, lo que indica una mejoría considerable en estos pacientes. Se concluyó que la prevalencia de leptospirosis está estrechamente relacionada con factores socioeconómicos como el saneamiento básico, el acceso a la información y una infraestructura adecuada en el lugar de residencia de las personas afectadas.
\end{abstract}

Palabras clave: Leptospirosis, región metropolitana, salud pública, zoonosis

\section{Introdução}

A leptospirose é uma zoonose de grande importância para saúde pública, é causada por uma bactéria aeróbica do gênero Leptospira, no qual até o momento foram identificados cerca de 200 sorovares em 14 diferentes espécies patogênicas (Brasil, 2014). A transmissão da Leptospira pode ocorrer através da urina de animais infectados, que contamina água ou lama, a bactéria ainda é capaz de penetrar pele e mucosas dos acometidos, lesionadas ou íntegras (Simões et al., 2016).

Segundo Adler \& Moctezuma (2010) a doença possuí uma distribuição mundial e maior incidência em locais de clima predominantemente tropical, ou seja, onde a ocorrência de elevados períodos de chuva e inundações é frequente. Além disso, conforme o estudo de Jesus et al. (2012), a associação desses fatores ambientais à precariedade do saneamento básico em áreas onde são observadas aglomerações e presença de roedores também são indícios que predispõem o aparecimento de novos surtos. No Brasil, a leptospirose é considerada uma doença endêmica e sua notificação às vigilâncias epidemiológicas é obrigatória (Llano, 2013).

No estado do Rio de Janeiro, de acordo com Alvares et al. (2013), a temperatura quente proporciona um clima favorável para o aparecimento de chuvas durante o ano, com destaque para uma maior incidência durante o verão, dessa forma, a região é constantemente categorizada como uma área epidêmica. Apenas em 2019, o estado teve 210 casos confirmados pelo SINAN (SINAN, 2019).

O objetivo desse estudo foi analisar os dados epidemiológicos referentes ao número de casos de leptospirose na região metropolitana I do estado do Rio de Janeiro entre 2015 e 2019. Por ser uma zoonose que provoca graves alterações, a leptospirose é uma doença de importância em saúde pública, e envolve questões socioeconômicas e ambientais, que interferem na qualidade de vida populacional e provoca um alto percentual de letalidade quando o devido protocolo não é aplicado.

\section{Material e métodos}

Foram analisados casos de leptospirose na região metropolitana I do estado do Rio de Janeiro cujas notificações foram feitas através do Sistema de Informação de Agravos de Notificação (SINAN) entre o ano de 2015 até o ano de 2019. A análise das notificações para leptospirose do presente artigo foi feita nos seguintes municípios da região metropolitana do estado do Rio de Janeiro: Rio de Janeiro, Duque de Caxias, Nova Iguaçu, São João de Meriti, Itaguaí, Belford Roxo, Magé, Mesquita, Seropédica, Queimados, Nilópolis e Japeri, e para definição do estudo epidemiológico foram selecionados como variáveis o gênero, faixa etária, escolaridade, município de notificação e o quadro de evolução da enfermidade nos indivíduos acometidos. 
Esses dados atualmente estão disponíveis na base de dados do departamento de informática do Sistema único de Saúde, conhecido como DATASUS. Foram considerados como casos suspeitos os indivíduos que apresentaram como sintomas clínicos febre, mialgia e cefaleia associados à sufusão conjuntival, sinais de insuficiência renal aguda, icterícia e/ou aumento de bilirrubinas e fenômeno hemorrágico. Além disso, também foram considerados como critérios a exposição a situações de risco nos 30 dias anteriores ao aparecimento dos primeiros sintomas. Os casos confirmados como positivos tiveram como auxílio o resultado de exames complementares laboratoriais feitos com reagentes para Leptospira (Sorologia IgM - Elisa, Micro aglutinação, Imuno-histoquímica e/ou RT-PCR).

Para a categorização dos dados obtidos no DATASUS-TABNET e geração dos gráficos do trabalho foi utilizado o programa Microsoft Excel.

\section{Resultados e discussão}

Na figura 1 são apresentados os números de casos notificados de leptospirose de acordo com o município de residência dos acometidos. Foi possível constatar uma alta prevalência da enfermidade na cidade do Rio de Janeiro, que isoladamente, contabilizou 248 casos, mais da metade dos casos confirmados entre 2015 a 2019 (56\%). Além disso, a Baixada Fluminense também apresentou um notável número de casos notificados, no qual os municípios de Duque de Caxias (14\%), Nova Iguaçu (9\%) e Belford Roxo (7\%) foram os que mais se destacaram nessa área da região metropolitana I do Rio de Janeiro.

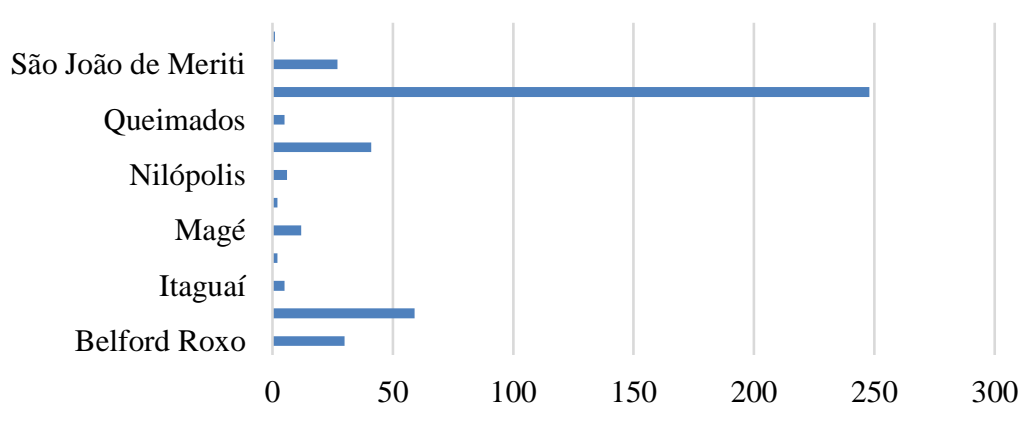

Figura 1. Distribuição de casos de leptospirose pelos municípios da região metropolitana I do estado do Rio de Janeiro entre os anos de 2015 a 2019 de acordo com os dados da plataforma DATASUS-TABNET.

Esses resultados, segundo Figueiredo et al. (2001) são decorrentes da disseminação da doença para o ambiente urbano nos últimos anos, no qual o deslocamento exorbitante de habitantes de áreas rurais para essas regiões mais populosas, muitas vezes para locais onde há precariedade e carência de infraestrutura e saneamento básico adequados, frequentemente tornam esses ambientes mais propensos a surtos de leptospirose devido a enchentes e a presença de possíveis roedores (Pelissari et al., 2011).

Um exemplo de migração populacional desordenada de áreas rurais para áreas urbanas são os chamados aglomerados subnormais, também conhecidos como favelas. De acordo com os dados do Instituto Brasileiro de Geografia e Estatística - IBGE (2020), estão comumente associadas aos grandes centros como Rio de Janeiro e São Paulo. Essas localidades por sofrerem deficiência na organização do espaço físico, frequentemente geram aglomerações de seus habitantes, excessivo acúmulo de lixo. De acordo com o estudo de Campos \& Santos (2019), consequentemente, descarte imprudente do mesmo em locais que não possuem saneamento básico adequado, como sistema eficiente de esgoto e também de coleta e descarte do lixo produzido. Fatores que fazem com que esses ambientes apresentem maiores índices de acometidos pela doença.

Quando esses aspectos socioeconômicos são relacionados à condições climáticas oportunas ao aparecimento de chuvas intensas, a incidência de enchentes e inundações nessas áreas são recorrentes, principalmente durante o verão, que segundo Velasco (2010), no qual o clima é úmido e de temperatura predominantemente quente, como acontece nos municípios do Rio de Janeiro e da Baixada Fluminense. Outra irrefutável causa para as possíveis enchentes nessas áreas, são as intervenções antrópicas, no qual 
o estudo de Costa et al. (2018) indicou que a migração em massa para áreas urbanas resultou na modificação desses locais, alternando o curso das águas pluviais e dificultando o escoamento, tendendo a ocasionar o acúmulo das mesmas.

Os dados analisados ainda apontam um menor índice de casos notificados em municípios como os de Seropédica, Mesquita e Japeri quando comparados ao de cidades mais populosas da região metropolitana do estado. Isso pode ocorrer por diversos fatores, como falta de conhecimento básico sobre os riscos que a leptospirose pode causar, dificuldade no acesso ao atendimento em postos de saúde, ou como sugere o estudo de Kogika et al. (1990), a subnotificação de casos pode acontecer devido a dúvida gerada durante o preenchimento das fichas e até pela falta de conhecimento da obrigatoriedade da notificação dos casos da doença por parte dos profissionais de saúde. Independente dos motivos, a subnotificação dos casos altera a coleta e captação dos dados, podendo subestimar drasticamente os resultados.

Na figura 2, os números de casos são apresentados de acordo com o gênero dos acometidos. É notável o maior grau de infecção em indivíduos do gênero masculino, o qual contabilizou um percentual de 87\% dos casos totais de leptospirose entre os anos de 2015 a 2019 na região metropolitana I do Rio de Janeiro. $\mathrm{O}$ percentual de casos de leptospirose em pessoas do gênero feminino foi de $13 \%$, prevalência muito menor quando comparada ao percentual de casos confirmados em homens.

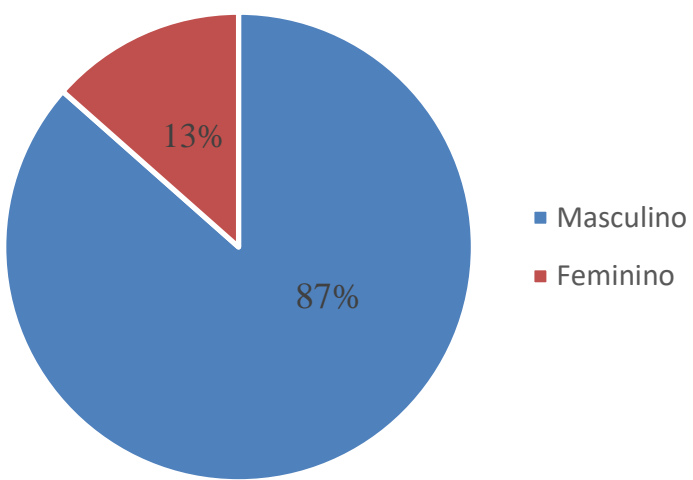

Figura 2. Percentual dos casos de leptospirose de acordo com o gênero dos acometidos baseado nos dados da plataforma DATASUS-TABNET.

A maior prevalência no acometimento de pessoas do gênero masculino é justificada por Vasconcelos et al. (2012) pela atuação dos homens em situações nas quais a exposição e consequente infecção pela leptospirose é maior, seja na coleta de lixo, manutenção de esgoto, e principalmente por esses indivíduos usualmente serem os mais procurados para ocuparem cargos onde a execução de atividades requeridas durante a ocorrências de enchentes, alagamentos e deslizamentos é essencial. Além disso, ainda é muito comum o maior desempenho de funções relacionadas ao gênero masculino em áreas rurais, onde a presença de roedores e outros animais que podem servir como reservatórios e transmissores da doença é habitual (Machado et al., 2017).

Na figura 3, o número de casos notificados de leptospirose é representado de acordo com a faixa etária dos acometidos, independente do gênero e do município de residência. Foi observado que o maior índice de casos positivos ocorreu em pessoas de 40 a 59 anos (40,9\%), seguido da faixa etária entre 20 a 39 anos $(33,3 \%)$, enquanto as faixas etárias com o menor percentual da doença foram as de crianças com menos de um até os quatro anos de idade $(0,45 \%)$ e em idosos de 80 anos ou mais $(0,45 \%)$.

A maior incidência de leptospirose em indivíduos entre 20 à 59 anos é justificada por Coelho et al. (2019) por essa ser a faixa etária na qual a população é ativa dentro do mercado de trabalho, fator que possibilita uma maior exposição a doença por estarem sujeitas a maiores situações de risco, facilitando a transmissão do patógeno entre elas.

Por outro lado, o menor acometimento de crianças até 4 anos e idosos foi corroborado pelos estudos de Carvalho et al. (2017) que sugeriram que esses indivíduos possuem contato restrito com áreas de solo e água contaminados pela leptospirose, especialmente durante época de enchentes e inundações, quando 
os acontecimentos de surtos da doença são mais propícios, pois essa população é comumente resgatada primeiro para locais seguros por serem considerados os membros mais frágeis da sociedade, diminuindo assim a sua contaminação.

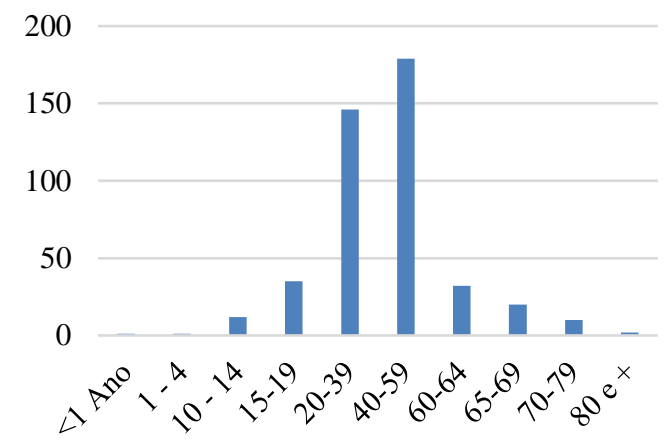

Figura 3. Número de casos de leptospirose de acordo com a faixa etária dos acometidos baseado nos dados da plataforma DATASUS-TABNET.

$\mathrm{Na}$ Figura 4 os dados são distribuídos de acordo com o grau de escolaridade dos acometidos. Entre as 438 ocorrências presentes na plataforma do SINAN entre 2015 à 2019 na região metropolitana I do Rio de Janeiro, foi observado um alto percentual de pacientes que optaram por não responder esse tópico na ficha de investigação, sendo a opção ignorado/branco a mais evidente, contabilizando 270 casos e atingindo um percentual de $61,6 \%$ das ocorrências totais.

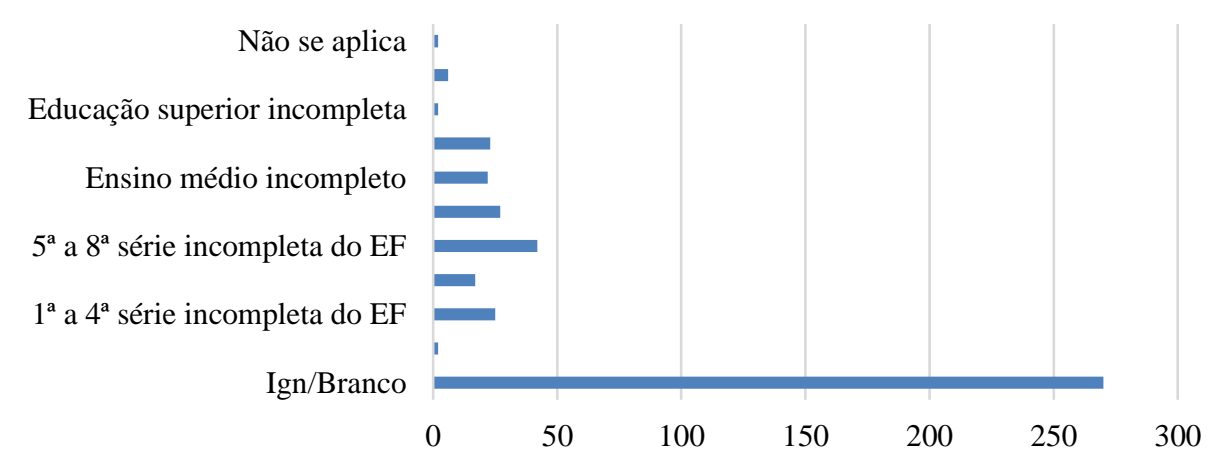

Figura 4. Número de casos de leptospirose de acordo com o grau de escolaridade dos acometidos baseado nos dados da plataforma DATASUS-TABNET.

O elevado percentual de casos preenchidos como ignorado/branco ocasiona uma notável alteração na análise dos dados coletados, fator que compromete os resultados obtidos. Isso pode ocorrer devido a inaptidão dos responsáveis pelo preenchimento dessas fichas nos postos de saúde, ou pelo constrangimento dos indivíduos acometidos pela doença em informar o grau de escolaridade, possivelmente por serem pessoas que possuem limitado acesso a informações e acesso a instituições de ensino básico.

Segundo Lermen \& Fisher (2010), a educação é o principal condutor de informações sobre a relação da saúde com o meio ambiente, com isso, as classes socioeconômicas e o nível de escolaridade de uma população são capazes de indicar e de se correlacionar com a morbidade de uma doença. Visto que a qualidade do ensino em escolas públicas é considerada baixa e usualmente é associada a pessoas de classes sociais mais pobres, a compreensão da preservação do meio no qual se vive acaba não ocorrendo, desse modo, o cuidado com fatores que predispõem a surtos de leptospirose é reduzido ou quase nulo (Mesquita et al., 2016).

Ainda de acordo com os estudos de Lermen \& Fisher (2010), os indivíduos que tem maior acesso a instituições de ensino e consequentemente maior grau de escolaridade, manifestam maior conhecimento da importância da conservação do meio onde vivem porém muitas vezes não possuem 
o senso de responsabilidade pela manutenção de áreas e locais públicos, indicando que em alguns casos o grau de escolaridade pode não interferir no número de acometidos, e a menor incidência dessa população pode estar associada a fatores como poder aquisitivo e a infraestrutura de suas respectivas moradias.

No quadro de evolução da leptospirose em pacientes da região metropolitana I do Rio de Janeiro entre 2015 a 2019 demonstrado na figura 5, foi possível observar que a maioria dos indivíduos apresentaram uma considerável melhora, contabilizando um percentual total de 67,3\% dos casos. Entretanto, 19,6\% dos acometidos foram à óbito pelo agravo da doença, e 12,6\% dos casos, que foram representados como ignorados/branco, não foram preenchidos na ficha de notificação.

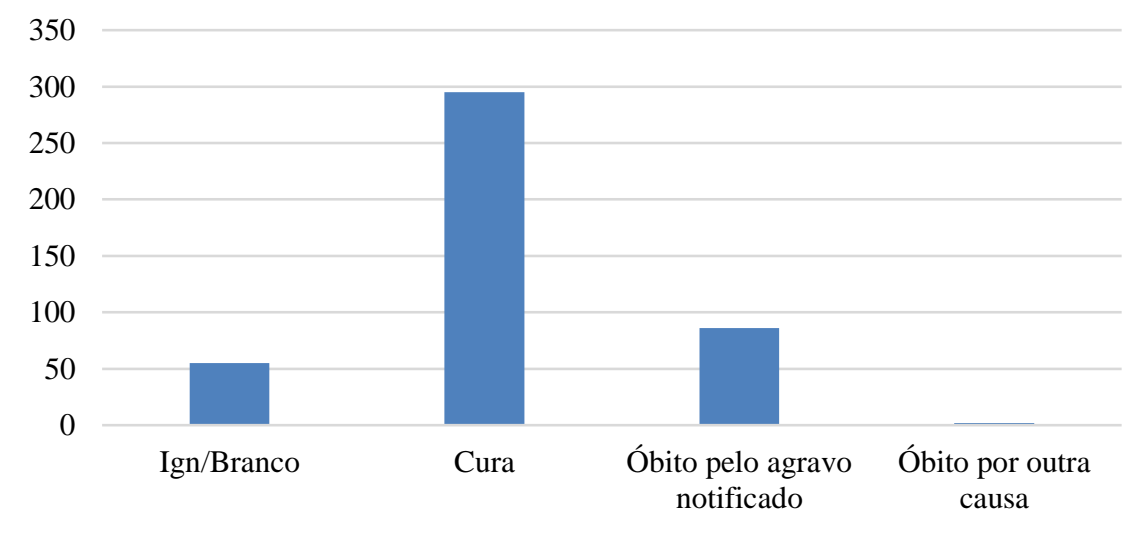

Figura 5. Número de casos de leptospirose de acordo com o quadro de evolução dos acometidos baseado nos dados da plataforma DATASUS-TABNET.

Alguns estudos como o de Lopes et al. (2004) sugerem que os indivíduos mais jovens desenvolvem os sintomas de menor gravidade quando comparados a indivíduos mais velhos. Dessa forma, o risco de mortalidade desse grupo diminui e geralmente esses pacientes têm um bom prognóstico, evoluindo para uma possível cura. Por outro lado, indivíduos com maior faixa etária tem maiores chances de evoluírem para o óbito visto que fazem parte do grupo comumente associado a doença renal crônica e outras graves doenças (Marinho et al., 2017), fatores muito agravantes que favorecem a evolução da doença.

A leptospirose, de acordo com o estudo de Ferreira et al. (2010), é uma doença classificada como bifásica, na qual a sua fase inicial é usualmente caracterizada por sintomas não específicos que podem ser confundidos com outras enfermidades (Chaiblich et al., 2017). Dessa forma, uma maior delonga na confirmação de um diagnóstico correto pode ainda gerar complicações mais graves como insuficiência renal e falência múltipla dos órgãos que ocasionam a morte do paciente acometido ( $\underline{\text { Sethi }}$ et al., 2010).

Os casos preenchidos como ignorados/branco usualmente indicam que houve falhas por parte dos responsáveis quanto ao preenchimento das fichas de notificação, seja devido a inaptidão dos profissionais em não ter a consciência sobre a importância da doença, ou pela interrupção abrupta do acompanhamento nos postos de saúde por parte do próprio paciente, onde a comunicação com o indivíduo muitas vezes é impossível de ser feita.

\section{Conclusão}

Diante dos resultados obtidos, pôde-se concluir que quando fatores ambientais facilitadores de enchentes e inundações são associados a importantes fatores socioeconômicos como infraestrutura inadequada e baixo poder aquisitivo, a possibilidade da ocorrência de novos surtos de leptospirose é muito mais alta. Considerando que a maioria dos acometidos não tem acesso a informações adequadas sobre a forma de transmissão da doença, geralmente devido ao baixo grau de escolaridade e por viverem em locais de maior incidência de leptospirose, seja pela expressiva migração popular para áreas que não suportam um grande número de pessoas ou por esses mesmos locais não possuírem serviços de saneamento básico adequados, a exposição a situações de risco de infecção é mais expressiva e 
alarmante. Dessa forma, é de extrema importância que sejam priorizadas a prevenção e conscientização em áreas onde as notificações são mais frequentes para que ocorra a regressão do número de casos, visto que as consequências causadas pela leptospirose são graves e afetam pessoas de diferentes faixas etárias e gênero, podendo levar os acometidos a óbito dependendo do grau de evolução da doença.

\section{Referências}

Adler, B., \& Moctezuma, A. P. (2010). Leptospira and leptospirosis. Veterinary Microbiology, 140(34), 287-296. https://doi.org/10.1007/978-1-0716-0459-5_24.

Alvares, C. A., Stape, J. L., Sentelhas, P. C., Moraes, G., Leonardo, J., \& Sparovek, G. (2013). Köppen's climate classification map for Brazil. Meteorologische Zeitschrift, 22(6), 711-728. https://doi.org/http://dx.doi.org/10.1127/0941-2948/2013/0507.

BRASIL, Ministério da Saúde. Departamento de informática do Sistema Único de Saúde-DATASUS. Leptospirose: Secção Rio de Janeiro. Disponível em: <http://tabnet.datasus.gov.br/cgi/deftohtm.exe?sinannet/cnv/leptorj.def>. Acesso em: 11 de setembro de 2020.

BRASIL. Ministério da Saúde. Gabinete do Ministro. Portaria n ${ }^{0}$ 1.461/GM/MS. Em 22 de dezembro de 1999. Lista Nacional de Doenças de Notificação Compulsória. Brasília: Ministério da Saúde, 2000. Doi: http://dx.doi.org/10.5123/S0104-16732000000100006

BRASIL. Ministério da Saúde. Leptospirose: Informações técnicas. Brasília. 2014. Disponível em: <https://www.saude.gov.br/saude-de-a-z/leptospirose/9729-informacoes-tecnicas〉. Acesso em: 02 de setembro de 2020.

Campos, F. L., \& Santos, R. A. (2019). Educação ambiental diante da problemática do lixo: uma análise descritiva em uma escola da área rural de Parnaíba - PI. Revista Ciências \& Ideias, 9(3), 80-93.

Carvalho, C. B. C., Gomes, M. L. C., Santos, C. L., Santos Rabello, R., \& Thomé, S. M. G. (2017). Leptospirose humana no estado do Rio de Janeiro: análise espaço-temporal e perfil dos casos confirmados no período de 2007 a 2014. Academus Revista Científica Da Saúde, 2(3), 10-22. https://doi.org/10.24118/reva1806.9495.2.3.2017:343.

Chaiblich, J. V., Lima, M. L. S., Oliveira, R. F., Monken, M., \& Penna, M. L. F. (2017). Estudo espacial de riscos à leptospirose no município do Rio de Janeiro (RJ). Saúde Em Debate, 41, 225-240. https://doi.org/10.1590/0103-11042017s219.

Coelho, A. G. V., Alves, I. J., \& Farias, V. L. V. (2019). Perfil epidemiológico dos casos de leptospirose na Região Metropolitana da Baixada Santista (SP), Brasil. BEPA, 16, 3-14.

Costa, A. J. S. T., Conceição, R. S., \& Amante, F. O. (2018). As enchentes urbanas e o crescimento da cidade do Rio de Janeiro: estudos em direção a uma cartografia das enchentes urbanas. Geo UERJ, $32,25685$.

Ferreira, T., Costa, V. C., \& Pereira, N. G. (2010). Diretrizes para diagnóstico e tratamento de leptospirose. In Serviço de Doenças Infecciosas e Parasitárias do Hospital Universitário Clementino Fraga Filho (pp. 1-10).

Figueiredo, C. M., Mourão, A. C., Oliveira, M. A., Alves, W. R., Ooteman, M. C., Chamone, C. B., \& Koury, M. C. (2001). Leptospirose humana no município de Belo Horizonte, Minas Gerais, Brasil: uma abordagem geográfica. Revista Da Sociedade Brasileira de Medicina Tropical, 34(4), 331-338. https://doi.org/10.1590/s0037-86822001000400004.

IBGE - Instituto Brasileiro de Geografia Estatística. Quase dois terços das favelas estão a menos de dois quilômetros de hospitais. 19 de mai. de 2020. Disponível em: <https://censo2021.ibge.gov.br/2012agencia-de-noticias/noticias/27728-quase-dois-tercos-das-favelas-estao-a-menos-de-doisquilometros-de-hospitais.html>. Acesso em 10 de outubro de 2020.

Jesus, M. S., Silva, L. A., Lima, K. M. da S., \& Fernandes, O. C. C. (2012). Cases distribution of leptospirosis in City of Manaus, State of Amazonas, Brazil, 2000-2010. Revista Da Sociedade Brasileira de Medicina Tropical, 45(6), 713-716. https://doi.org/10.1590/s003786822012000600011.

Kogika, M. M., Hagiwara, M. K., \& Mirandola, R. M. S. (1990). Alterações bioquímicas na leptospirose 
canina. Brazilian Journal of Veterinary Research and Animal Science, 27(2), 177-182. https://doi.org/10.11606/issn.0000-0000.272177-182.

Lermen, H. S., \& Fisher, P. D. (2010). Percepção ambiental como fator de saúde pública em área de vulnerabilidade social no Brasil. Revista de APS, 13(1), 62-71.

Llano, H. A. B. (2013). Revisão e situação atual da brucelose e leptospirose em bovinos no Brasil e na Colômbia. Seminário Apresentado Junto à Disciplina Seminários Aplicados Do Programa de PósGraduação Em Ciência Animal Da Escola de Veterinária e Zootecnia Da Universidade Federal de Goiás. Goiânia.

Lopes, A. A., Costa, E., Costa, Y. A., Sacramento, E., Oliveira Junior, A. R. R., Lopes, M. B., \& Lopes, G. B. (2004). Comparative study of the in-hospital case-fatality rate of leptospirosis between pediatric and adult patients of different age groups. Revista Do Instituto de Medicina Tropical de Sao Paulo, 46(1), 19-24. https://doi.org/10.1590/S0036-46652004000100004.

Machado, G. B., Neto, A. C. S., Dewes, C., Fortes, T. P., Pacheco, P. S., Freitas, L. S., Felix, S. R., \& Silva, É. F. (2017). Leptospirose Humana: uma revisão sobre a doença e os fatores de risco associados à zona rural. Science And Animal Health, 5(3), 238-250. https://doi.org/10.15210/sah.v5i3.11412.

Marinho, A. W. G. B., Penha, A. P., Silva, M. T., \& Galvão, T. F. (2017). Prevalence of chronic renal disease among Brazilian adults: a systematic review. Cadernos Saúde Coletiva, 25(3), 379-388. https://doi.org/10.1590/1414-462x201700030134.

Mesquita, M. O., Trevilato, G. C., Saraiva, L. H., Schons, M. S., \& Garcia, M. I. F. (2016). Material de educação ambiental como estratégia de prevenção da leptospirose para uma comunidade urbana reassentada. Cadernos Saúde Coletiva, 24(1), 77-83. https://doi.org/10.1590/1414462x2016000x0428.

Pelissari, D. M., Maia-Elkhoury, A. N. S., Arsky, M. de L. N. S., \& Nunes, M. L. (2011). Revisão sistemática dos fatores associados à leptospirose no Brasil, 2000-2009. Epidemiologia e Serviços de Saúde, 20(4), 565-574. https://doi.org/10.5123/S1679-49742011000400016.

Sethi, S., Sharma, N., Kakkar, N., Taneja, J., Chatterjee, S. S., Banga, S. S., \& Sharma, M. (2010). Increasing trends of leptospirosis in northern India: a clinico-epidemiological study. PLoS Neglected Tropical Diseases, 4(1), e579. https://doi.org/10.1371/journal.pntd.0000579.

Simões, L. S., Sasahara, T. H. C., Favaron, P. O., \& Miglino, M. A. (2016). Leptospirose-Revisão. PUBVET, 10, 111-189. https://doi.org/10.22256/pubvet.v10n2.138-146.

SINAN, 2019

Vasconcelos, C. H., Fonseca, F. R., Lise, M. L. Z., \& Arsky, M. (2012). Fatores ambientais e socioeconômicos relacionados à distribuição de casos de leptospirose no Estado de Pernambuco, Brasil, 2001-2009. Caderno de Saúde Coletiva, 20(1), 49-56.

Velasco, K. S. (2010). Impacto das enchentes nos municípios da Baixada Fluminense. Universidade Candido Mendes, Rio de Janeiro.

Histórico do artigo:

Recebido: 7 de dezembro de 2020.

Aprovado: 17 de janeiro de 2021
Licenciamento: Este artigoé publicado na modalidade Acesso Aberto sob a licença Creative Commons Atribuição 4.0 (CC-BY 4.0), a qual permite uso irrestrito, distribuição, reprodução em qualquer meio, desde que $\mathrm{o}$ autor e a fonte sejam devidamente creditados. 